\title{
EcoRe3 - Resistance, Recovery and Resilience of Long- term Ecological Systems
}

\author{
Alistair W.R. Seddon', L.E. Cole ${ }^{2}$, J. Morris ${ }^{3}$, M. Shawn-Fletcher ${ }^{4}$ and K.J. Willis ${ }^{5}$
}

\begin{abstract}
"Resilience" is a key attribute needed to ensure the persistence of Earth's ecosystems in the face of increasing anthropogenic stressors and climate change. Yet definitions of resilience, and the methods used to measure it, can differ markedly between studies (Hodgson et al. 2015). These disagreements make it difficult to compare and identify systems with more or less resilience and to plan future mitigation strategies.
\end{abstract}

One useful approach is to integrate the different concepts of resilience within one unifying framework, by identifying the different "components" of resilience that lead to a system maintaining ecological functioning in the face of disturbance (Fig. 1). This is the first step for understanding what factors cause variations in resilience between different ecosystems. The next challenge is to develop tools to measure these components across biomes.

Recent attempts have used satellite data to map some components of resilience at global scales (e.g. Seddon et al. 2016). These "snapshots" are based on measuring shortterm ecological responses, but whether the patterns reflect fundamental properties of the systems, or are the result of historical disturbance legacies, remains unknown. Sediments provide a long-term record of disturbances and ecological responses for investigating resilience (e.g. Cole et al. 2014),
Elasticity: Speed of recovery to equilibrium following a disturbance. Determines the recovery rate.

Precariousness: Distance to an ecological threshold or tipping point.

Latitude: Width of the basin (or ecological resilience, e.g. Holling 1973)

Resistance: Amount of change following a disturbance (in Fig. 1, basin with slope "a" has higher resistance than slope "b"; e.g. de Keersmaeker et al. 2015).

Sensitivity: Relative variance of system compared to driver (e.g. Seddon et al. 2016).

Box 1: Components of resilience - definitions

but the appropriate metrics for comparing components of resilience from sediments across different ecosystem types are yet to be developed.

EcoRe 3 is a new PAGES working group which aims to devise a set of standardized approaches for comparing components of resilience from the paleo-record. We focus on measuring resistance (the amount of change following a disturbance), recovery (the speed to return to equilibrium following a disturbance) and how these components contribute to the resilience (the ability to tolerate

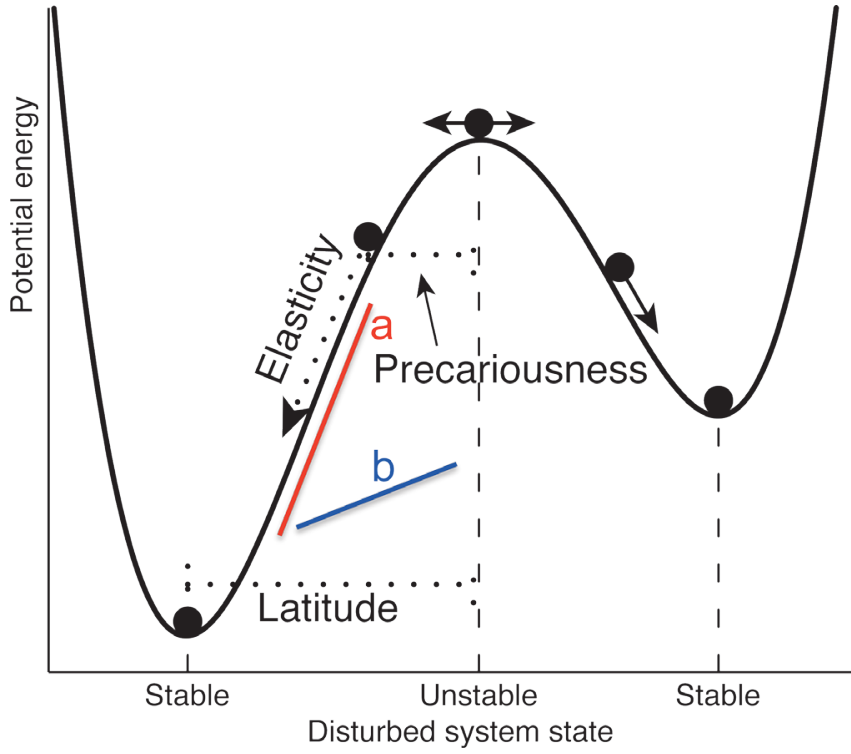

Figure 1: Components of resilience (adapted from Hodgson et al. 2015), showing a classical resilience landscape where components of resilience can be derived. See Box 1 for definitions. disturbance and remain in the same state) in ecological systems using long-term data.

\section{Scientific objectives}

EcoRe 3 is based around four key scientific objectives:

Theory: We will develop methods, grounded in ecological theory, to measure and compare resistance, recovery and resilience using long-term ecological datasets. These will be explored at the first EcoRe 3 workshop in March 2017 (www.pastglobalchanges.org/calendar/127-pages/1652-ecore3-1st-wshop-17).

Application: Using a series of high-resolution paleoecological records to test the use of these approaches will enable us to compare components of resilience across biomes, and investigate which biotic and abiotic controls are responsible for the observed patterns.

Training: Open-Source code will be developed for all analyses to ensure reproducibility of the methods. A workshop for early-career scientists, towards the end of the group's third year, is planned.

Integration: A major goal is to find ways to collaborate with scientists interested in resilience across different disciplines. In addition to working together with other PAGES working groups, we plan to investigate how to integrate the tools developed on paleo-datasets into those that can be used for the management of contemporary ecosystems.

For further information about EcoRe3 activities, please subscribe to the mailing list. http://pastglobalchanges.org/ini/wg/ecore3/

\section{AFFILIATIONS}

'Department of Biology, University of Bergen, Norway 2Department of Zoology, University of Oxford, UK ${ }^{3}$ Department of Geography, University of Utah, Salt Lake City, USA

${ }^{4}$ School of Geography, University of Melbourne, Australia

${ }^{5}$ Oxford University and Royal Botanic Gardens, Oxford, UK

\section{CONTACT}

Alistair Seddon: alistair.seddon@uib.no

\section{REFERENCES}

Cole LES et al. (2014) Nature Comm 5, doi:10.1038/ ncomms 4906

Hodgson D et al. (2015) Trends Ecol Evol 30: 503-506 Holling CS (1973) Annu Rev Ecol Evol Syst 4: 1-23

Seddon AWR et al. (2016) Nature 531: 229-232 